\title{
Important Role of Tissue Angiotensin-converting Enzyme Activity in the Pathogenesis of Coronary Vascular and Myocardial Structural Changes Induced by Long-Term Blockade of Nitric Oxide Synthesis in Rats
}

\author{
Masao Takemoto, ${ }^{\star}$ Kensuke Egashira, ${ }^{*}$ Makoto Usui, ${ }^{\star}$ Kohtaro Numaguchi, ${ }^{\star}$ Hideharu Tomita, ${ }^{*}$ Hiroyuki Tsutsui, \\ Hiroaki Shimokawa, ${ }^{*}$ Katsuo Sueishi, ${ }^{\ddagger}$ and Akira Takeshita* \\ * Research Institute of Angiocardiology and Cardiovascular Clinic, and ${ }^{\ddagger}$ First Department of Pathology, Kyushu University School of \\ Medicine, Fukuoka, Japan
}

\begin{abstract}
The long-term administration of $N^{\omega}$-nitro-L-arginine methyl ester (L-NAME), an inhibitor of nitric oxide synthesis, produces coronary vascular remodeling and myocardial hypertrophy in animals. This study used a rat model to investigate the role of angiotensin I converting enzyme (ACE) in the pathogenesis of such changes. We studied the following groups, all of which received drug treatment in their drinking water: untreated controls, and those administered L-NAME, L-NAME, and an ACE inhibitor (ACEI), and L-NAME and hydralazine. Cardiovascular structural changes and tissue ACE activities were evaluated after the first, fourth, and eighth week of treatment. In rats treated with L-NAME alone, vascular remodeling was evident at the fourth and eighth week, and myocardial hypertrophy was present at the eighth week of treatment. The vascular and myocardial remodeling were characterized by increased tissue ACE activities and immunodetectable ACE in those tissues. These changes were markedly reduced by ACEI, but not by hydralazine treatment. Increased local ACE expression may thus be important in the pathogenesis of cardiovascular remodeling in this model. (J. Clin. Invest. 1997. 99: 278-287.) Key words: angiotensin converting enzyme inhibitor $\bullet$ renin-angiotensin system $\bullet$ nitric oxide $\bullet$ left ventricular hypertrophy $\bullet$ coronary circulation
\end{abstract}

\section{Introduction}

The endothelium is important in maintaining the homeostasis of the blood vessel wall by producing endothelium-derived substances (1-2). An important endothelium-derived relaxing factor has been identified as nitric oxide $(\mathrm{NO}),{ }^{1}$ or a related

Address correspondence to Kensuke Egashira, M.D., Ph.D. Research Institute of Angiocardiology Cardiovascular Clinic, Kyushu University School of Medicine, 3-1-1, Maidashi, Higashi-ku, Fukuoka, 81282, Japan. Phone: 81-92-641-1151 x2306; FAX: 81-92-633-2742.

Received for publication 2 August 1996 and accepted in revised form 14 November 1996.

1. Abbreviations used in this paper: $\mathrm{ACE}$, angiotensin I converting enzyme; ACEI, angiotensin I converting enzyme inhibitor; Hyd, hydralazine; L-NAME, $N^{\omega}$-nitro-L-arginine methyl ester; LV, left ventricle; NO, nitric oxide; PRA, plasma renin activity.

J. Clin. Invest.

(C) The American Society for Clinical Investigation, Inc.

0021-9738/97/01/278/10 \$2.00

Volume 99, Number 2, January 1997, 278-287 compound $(3,4)$. Besides its role in controlling vascular tone, platelet aggregation, and leukocyte adhesion, extensive evidence suggests that NO inhibits the growth and/or proliferation of blood vessels (5-9). Studies in animals and humans have shown that disorders such as hypercholesterolemia and atherosclerosis are associated with dysfunction of the endothelium of the large epicardial and resistance coronary arteries (10-13). Endothelial dysfunction may lead to functional and structural abnormalities in the vascular wall, thus contributing to myocardial ischemia.

We (14-16) and other investigators (17-21) reported recently that long-term blockade of NO synthesis by the chronic administration of $N^{\omega}$-nitro-L-arginine methyl ester (L-NAME), an inhibitor of NO synthesis, produces systemic arterial hypertension, microvascular structural changes with thickening of the media, luminal narrowing, perivascular fibrosis, and hypertrophy of the myocardium in rats and pigs. Evidence suggests that NO synthesis is actually reduced in these animals (17-19). These observations indicate that the coronary vascular and myocardial structural changes are due to a defect in the NO synthesis that leads to the activation of neurohormonal systems and growth-promoting factors, not due to the presence of systemic arterial hypertension per se. However, the exact pathogenesis of these cardiovascular structural changes is unclear.

Reports suggest that the renin-angiotensin system is activated in this model of chronic administration of L-NAME. The chronic administration of L-NAME increases plasma renin activity (PRA) and cardiac tissue angiotensin I converting enzyme (ACE) activity (17-21). It has been suggested that ACE activation contributes to the development of vascular and myocardial structural changes after left ventricular hypertrophy and failure in rats (22-26). ACE inhibitors (ACEI) prevent cardiac remodeling and prolongs survival after myocardial infarction in both animals (24-26) and humans $(27,28)$. Such evidence suggests that the inhibition of activated ACE may explain the benefits of ACEI in those conditions. However, it has not been determined whether ACE activation is involved in the pathogenesis of the vascular and myocardial structural changes in the rat model that is chronically administered L-NAME.

The purpose of this study was to investigate the role of ACE in the pathogenesis of vascular and myocardial structural changes in rats induced by long-term administration of the NO synthase inhibitor, L-NAME. We evaluated the effects of ACEI on the cardiovascular structural changes and tissue ACE activities in this model. In addition, we used immunohistochemistry to determine the local tissue expression of ACE under some of these conditions. 


\section{Methods}

These experiments were reviewed and approved by the Committee on Ethics of Animal Experiments, Faculty of Medicine, Kyushu University, and conducted according to the Guidelines for Animal Experiments of the Faculty of Medicine, Kyushu University and Law (No. 105) and Notification (No. 6) of the Japanese Government.

Drugs and antibodies. This study used L-NAME (Sigma Chemical Co., St. Louis, MO), temocapril (Sankyo Pharmaceutical Co., Tokyo, Japan), and hydralazine (Ciba-Geigy Pharmaceutical Co., Tokyo, Japan). An antibody for rat ACE (9B9) was from Immunobiology Lab, (Fujioka, Gunma, Japan).

Animal preparation. 191 male Wistar-Kyoto rats 20-wk old were obtained from an established colony at the Animal Research Institution of Kyushu University School of Medicine. Six groups of rats were studied. The control group received untreated drinking water. The second group (L) received L-NAME in its drinking water $(1 \mathrm{mg} / \mathrm{ml})$. At this concentration, the daily intake of L-NAME for the latter group was $\sim 30-40 \mathrm{mg} / \mathrm{d}$. The third group (L+ACEI1) received L-NAME and angiotensin-converting enzyme inhibitor (temocapril, $0.1 \mathrm{mg} / \mathrm{ml}$ ) in drinking water; the fourth group (L+ACEI2) received L-NAME and temocapril $(0.01 \mathrm{mg} / \mathrm{ml})$; the fifth group (L+Hyd) L-NAME and hydralazine $(0.12 \mathrm{mg} / \mathrm{ml})$; and the sixth group (ACEI) temocapril alone $(0.01 \mathrm{mg} / \mathrm{ml})$ in the drinking water. All rats were fed a normal diet and single-housed in a special pyrogen-free facility. We measured the actual volume of water drunk by each group of rats on a weekly basis and confirmed that the animals all got $30-40 \mathrm{ml}$ of the water containing drugs.

Systolic blood pressure (the tail-cuff method) and body weight were measured every week. At the first and fourth weeks of treatment, morphometric and biochemical analyses were performed in the control, L, and L+ACEI1 groups. At the eighth week of treatment, those analyses were performed in the rats of all six groups that survived the full 8 wk of treatment. All rats were anesthetized by thiopentobarbital, and then killed by exsanguination.

Histopathology and morphometry. Histopathology and morphometry were performed by investigators who were blind to the treatment being administered. Findings were evaluated in 10-15 rats of each group as previously described (15). Excised hearts were perfused with physiological saline solution and fixed with $6 \%$ formaldehyde solution for $30 \mathrm{~min}$ via retrograde infusion into the descending aorta at a pressure of $90 \mathrm{mmHg}$. The left ventricle (LV) was separated from the atria, the right ventricle, and the great vessels. The LV was weighed and cut into five pieces perpendicular to the long axis. The thoracic aorta was also excised. Tissues were fixed in $6 \%$ formaldehyde for a few days, then dehydrated and embedded in paraffin. The paraffin slices were stained with hematoxylin and eosin and Masson's trichrome staining solutions. The whole area of all histopathologic sections was scanned using a light microscope (Microphot-FXA, Nikon Co., Tokyo, Japan) equipped with a two-dimensional analysis system.

To evaluate the thickening of the coronary arterial wall and perivascular fibrosis, short-axis images of the large coronary arteries (internal diameters $\geq 200 \mu \mathrm{m}$ ) and small coronary arteries (internal diameters $<200 \mu \mathrm{m}$ ) were studied. The inner border of the lumen and the outer border of the tunica media were traced in each arterial image with Masson's trichrome staining at magnifications of 100-200. Areas encircled by the tracings were calculated. During the quantification procedure, nonround vessels due to oblique transsection or branching were excluded, and only round vessels were studied. The wall-to-lumen ratio (the medial thickness to the internal diameter) and the area of fibrosis (collagen deposition stained with aniline blue) immediately surrounding the blood vessels were then calculated. Perivascular fibrosis was determined as the ratio of the area of fibrosis surrounding the vessel wall to the total vessel area. In each heart, $\sim 40$ small arteries and 10 large arteries were examined. Average values for each size of vessel were used for analysis. The wall-to-lumen ratio of the thoracic aorta was examined similarly.

To measure the myocyte cross-sectional area, morphometry of left ventricular myocytes was performed $(29,30)$. In Masson's trichromestained section of the lateral mid-free wall of the LV, the myocyte cross-sectional area was measured from myocytes that were cut transversely and had both a visible nucleus and an unbroken cellular membrane. The outer borders of the myocytes were traced and the myocyte areas were calculated. Approximately 100 cells were counted per heart and the average was used for analysis.

Biochemical analysis. Biochemical parameters were measured in the other 5-10 rats of each group. Animals were anesthetized with pentobarbital and blood samples were taken from the femoral artery. The following parameters were measured at the first, fourth, and eighth week of treatment: PRA and ACE activity of the serum and heart. At the eighth week of treatment, ACE activities of lung, thoracic aorta, lung, kidney, and liver were also measured. PRA was determined by radioimmunoassay using commercially available kits. PRA values are expressed as nanograms of angiotensin I synthesized per milliliter/hour. Serum and tissue ACE activities were determined

Table I. Systolic Blood Pressure, Heart Rate, and Body Weight

\begin{tabular}{|c|c|c|c|c|c|c|c|}
\hline Parameter & Week & Control & $\begin{array}{l}\text { L-NAME } \\
\text { alone }\end{array}$ & $\begin{array}{l}\text { L-NAME } \\
+ \text { Hyd }\end{array}$ & $\begin{array}{l}\text { L-NAME } \\
+ \text { ACEI1 }\end{array}$ & $\begin{array}{l}\text { L-NAME } \\
+ \text { ACEI2 }\end{array}$ & $\begin{array}{l}\text { ACEI } \\
\text { alone }\end{array}$ \\
\hline & & $(n=40)$ & $(n=60)$ & $(n=20)$ & $(n=45)$ & $(n=16)$ & $(n=10)$ \\
\hline \multirow[t]{4}{*}{ Systolic blood pressure (mmHg) } & 0 & $133 \pm 3$ & $133 \pm 3$ & $130 \pm 2$ & $132 \pm 3$ & $133 \pm 3$ & $134 \pm 3$ \\
\hline & 1 & $135 \pm 2$ & $158 \pm 3$ & NM & $140 \pm 3$ & NM & NM \\
\hline & 4 & $136 \pm 5$ & $175 \pm 4 *+$ & NM & $137 \pm 5$ & NM & NM \\
\hline & 8 & $134 \pm 2$ & $188 \pm 4 * *$ & $143 \pm 6$ & $134 \pm 4$ & $193 \pm 5 * \neq$ & $131 \pm 5$ \\
\hline \multirow[t]{4}{*}{ Heart rate $(\mathrm{bpm})$} & 0 & $418 \pm 8$ & $413 \pm 6$ & $417 \pm 7$ & $416 \pm 8$ & $421 \pm 7$ & $406 \pm 3$ \\
\hline & 1 & $426 \pm 7$ & $355 \pm 13$ & NM & $377 \pm 14$ & NM & NM \\
\hline & 4 & $418 \pm 10$ & $391 \pm 6$ & NM & $385 \pm 16$ & NM & NM \\
\hline & 8 & $418 \pm 5$ & $412 \pm 6$ & $410 \pm 11$ & $404 \pm 9$ & $399 \pm 9$ & $428 \pm 6$ \\
\hline \multirow[t]{4}{*}{ Body weight (g) } & 0 & $333 \pm 5$ & $336 \pm 4$ & $328 \pm 4$ & $336 \pm 7$ & $330 \pm 6$ & $330 \pm 5$ \\
\hline & 1 & $346 \pm 7$ & $322 \pm 6$ & $\mathrm{NM}$ & $328 \pm 7$ & NM & NM \\
\hline & 4 & $358 \pm 9$ & $328 \pm 7$ & NM & $317 \pm 9$ & NM & NM \\
\hline & 8 & $381 \pm 8 *$ & $286 \pm 6^{* \pm}$ & $332 \pm 6^{\ddagger}$ & $324 \pm 5^{\ddagger}$ & $310 \pm 6^{* *}$ & $363 \pm 5^{*}$ \\
\hline
\end{tabular}

Values are mean \pm SEM. $* P<0.01$ versus the 0 week; ${ }^{*} P<0.01$ versus the control group; NM, not measured. 


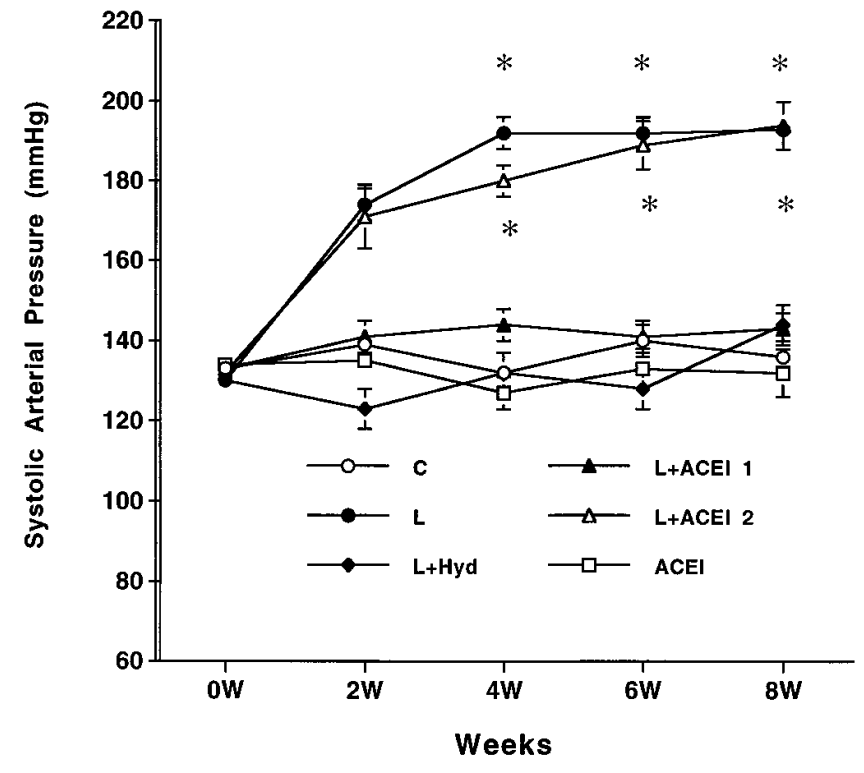

Figure 1. Time-related changes in systolic arterial pressure in the $\mathrm{C}$ (control), L (L-NAME alone), L+Hyd (L-NAME+hydralazine), L+ ACEI1 (L-NAME + high dose ACE inhibitor), L+ACEI2 (LNAME+ low dose ACE inhibitor), and ACEI (ACEI alone) groups. $* P<0.01$ versus the $\mathrm{C}$ group. Values are mean \pm SEM.

using a fluorometric assay that was described by Cheung and Cushman (31). Serum ACE activity was calculated as nanomoles of HisLeu generated per milliliter of serum/hour, and tissue ACE activity was calculated as the nanomoles His-Leu generated per milligram tissue weight of serum/hour (32).

Immunohistochemistry. Immunohistochemistry was performed at the fourth and eighth week of treatment on the cardiac tissues from five rats each from the control, L, L+ACEI1, and L+Hyd groups. After the hearts were perfused with physiological saline without pressure fixation, tissue samples were embedded immediately in OCT compound, frozen, cut into 4-mm-thick sections, and mounted on glass slides. After rehydration, the sections were preincubated with $0.1 \%$ Triton X-100 in PBS and $0.1 \%$ skim milk to decrease nonspecific binding. An antibody to rat ACE was applied and the slides were incubated for $60 \mathrm{~min}$ at room temperature. The sections were incubated with biotinylated rabbit anti-mouse immunoglobulin ( $\mathrm{IgG}$ ) for $10 \mathrm{~min}$ and then incubated with $1 \%$ hydrogen peroxide $\left(\mathrm{H}_{2} \mathrm{O}_{2}\right)$ in methanol to quench endogenous peroxidase. The sections were then incubated with peroxidase-labeled streptavidin solution for $10 \mathrm{~min}$. The slides were rinsed in PBS with $0.1 \%$ Triton X-100 after each incubation step. The peroxidase activity was identified by the use of DAB-buffer tablets (Merck 64271) with $\mathrm{H}_{2} \mathrm{O}_{2}(0.013 \%)$. The sections were counterstained with hematoxylin solution, dehydrated in ethanol, and then mounted. The degree of the ACE immunoreactivity in the coronary arteries and myocardium was evaluated by two independent data-blind observers.

Statistical analysis. Data are expressed as the mean \pm SEM. Paired data were compared by Student's $t$ tests. Myocyte size, heart weight, body weight, and hemodynamic parameters were compared by using one-way ANOVA followed by Bonferroni's test for multiple comparisons. Comparisons of vascular wall-to-lumen ratio, perivascular fibrosis, and time-related changes in blood pressure among groups were performed using two-way ANOVA followed by Bonferroni's multiple-comparison $t$ tests. Correlations between the two parameters were determined by simple linear regression analysis. $P<0.05$ was considered statistically significant.
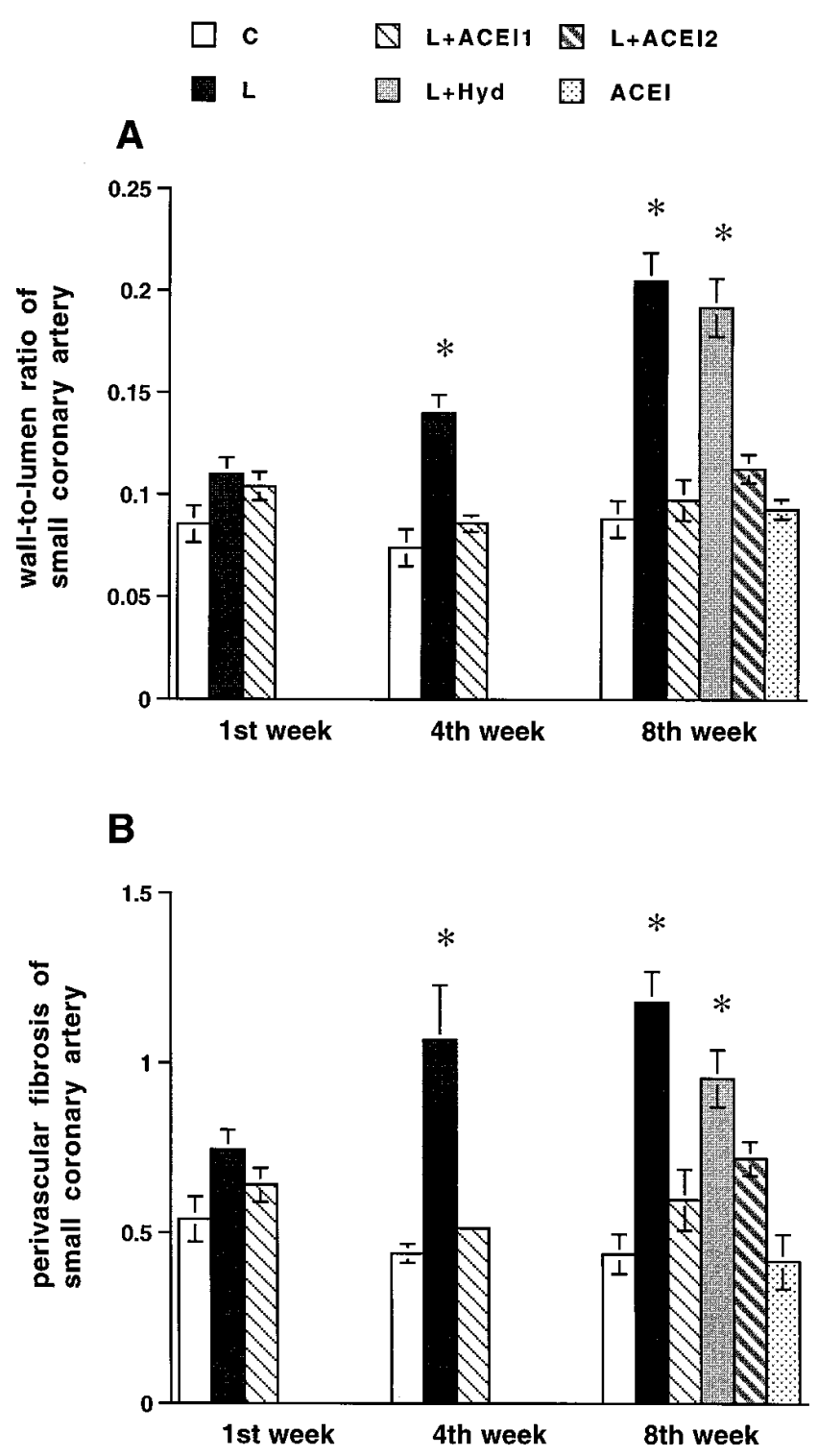

Figure 2. The wall-to-lumen ratio in small coronary arteries at the first, fourth, and eighth week of treatment $(A)$ and perivascular fibrosis in small coronary arteries at the first, fourth, and eighth week of treatment $(B)$ in the control, $\mathrm{L}, \mathrm{L}+\mathrm{Hyd}, \mathrm{L}+\mathrm{ACEI} 1, \mathrm{~L}+\mathrm{ACEI}$, and ACEI groups. $* P<0.01$ versus the $\mathrm{C}$ group by two-way ANOVA and multiple comparison $t$ tests.

\section{Results}

Body weight and hemodynamic variables. Body weights before the treatments were identical among groups (Table I). Rats in the control and ACEI groups gained weight, whereas those in the $\mathrm{L}$ and $\mathrm{L}+\mathrm{ACEI} 2$ groups lost weight during the study. Body weights did not change significantly in the $\mathrm{L}+\mathrm{Hyd}$ and L+ACEI1 groups during the study.

Time-related changes in systolic arterial pressure are shown in Fig. 1. In the L group, systolic arterial pressure increased progressively and plateaued after $4 \mathrm{wk}$ of treatment. The increases in systolic arterial pressure in the L+ACEI2 group resembled those in the L group. No significant changes in systolic arterial pressure were observed in the control, 

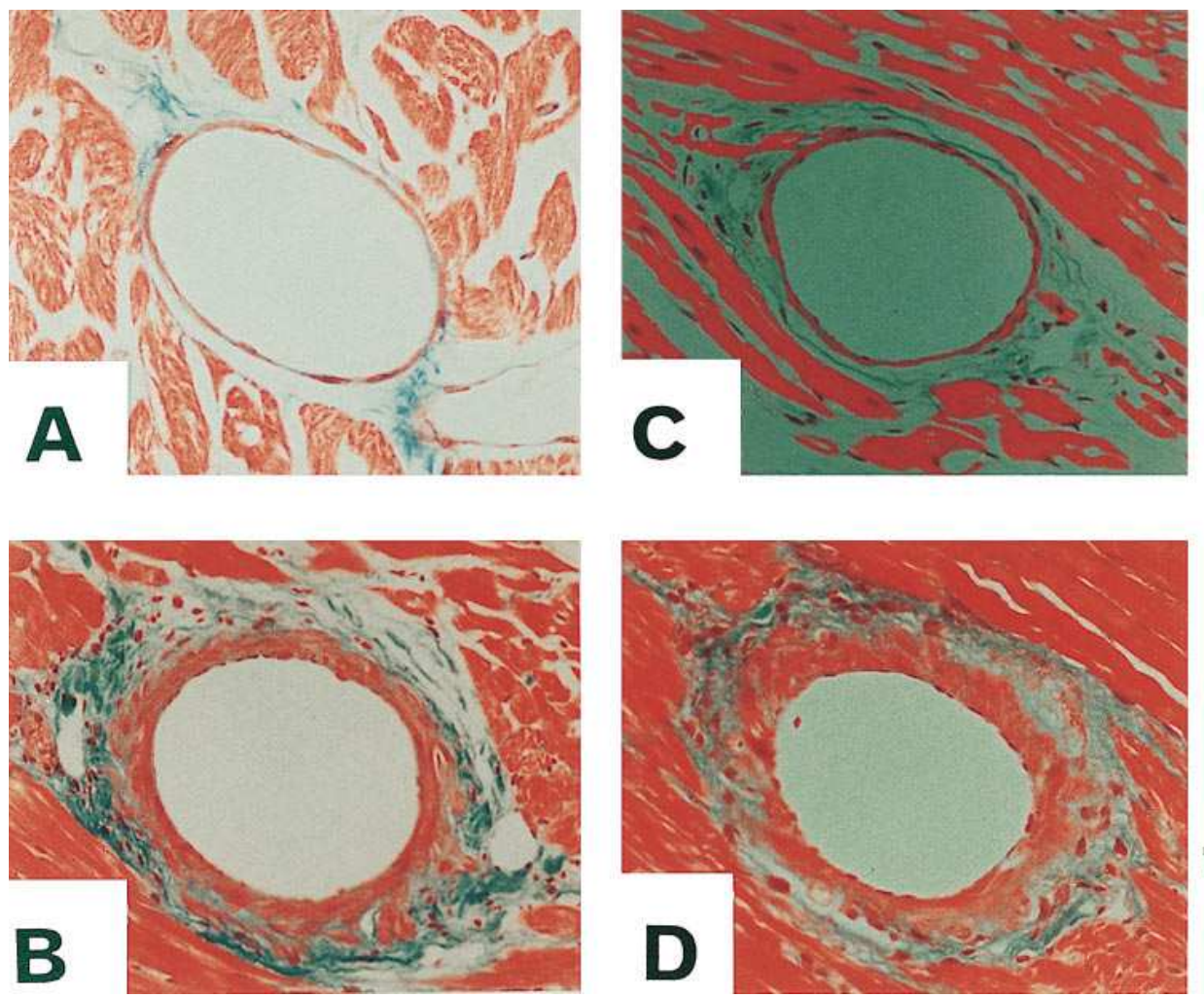

\section{$100 \mu \mathrm{m}$}

Figure 3. Micrographs of small coronary arteries with Masson's trichrome stain in the control $(A), \mathrm{L}(B)$, L-NAME + ACEI $(C)$ and L-NAME + Hyd $(D)$ groups. A bar indicates $100 \mu \mathrm{m}$.

L + Hyd, L+ACEI1, and ACEI groups. Heart rate was similar throughout the treatment among the six groups (Table I).

Wall-to-lumen ratio and perivascular fibrosis. The wallto-lumen ratios and the degree of perivascular fibrosis in the small coronary arteries were examined after the first and fourth week of treatment in three (control, L, and L+ACEI1) groups. These two parameters did not change after the first week of treatment in any of these groups. However, after the fourth week of treatment, they were significantly greater in the L group than in the control group (Fig. 2). These vascular structural changes were reduced by treatment with ACEI.

The wall-to-lumen ratios and perivascular fibrosis in the small coronary arteries were examined after the eighth week of treatment in all six groups. These parameters were significantly greater in the L group than in the control group (Fig. 2). These vascular structural changes were significantly reduced by treatment with either a low or high dose of ACEI, but not by hydralazine. Microscopic pictures taken of small coronary arteries with Masson's trichrome stain for the control, L, L+ACEI1, and L+hydralazine (Hyd) groups are shown in Fig. 3. Treatment with ACEI alone had no effect on the coronary vascular structure.

The wall-to-lumen ratios in the large coronary arteries and thoracic aorta were also examined after the eighth week of treatment. This parameter was slightly, but significantly, greater in the L group than in the control group (Fig. 4). The structural changes in the large coronary arteries and aorta were significantly reduced by the low and high doses of ACEI, but not by hydralazine treatment. Treatment with ACEI alone had no effect on structure in the large epicardial coronary ar- teries and aorta. There were no significant differences in the degree of perivascular fibrosis of the large coronary arteries among the six groups (Fig. 4).

Myocardial hypertrophy. At the first and fourth week of treatment, the relative weights of the LV and the cross-sectional areas of LV myocytes did not significantly differ among the control, L, and L+ACEI1 groups. At the eighth week of treatment, these parameters were increased in the $\mathrm{L}$ group compared with controls (Figs. 5 and 6). The increases in the relative $\mathrm{LV}$ weights and myocyte cross-sectional areas in the $\mathrm{L}$ group were blunted to a similar extent by both the low and high doses of ACEI, but were unaffected by hydralazine. The myocyte areas in the ACEI group did not significantly differ from those of the control group.

Biochemical data and immunohistochemistry. PRA and ACE activities are shown in Table II. PRA in the control group is somewhat higher than that of normal animals in other studies. This was because animals were anesthetized for blood sampling in this study. Compared with the control group, PRA in the L group did not change at the first or the fourth week, and significantly increased after the eighth week of treatment. The increases in PRA seen in the L group at the eighth week of treatment were also observed in the L+Hyd, L+ACEI1, L+ACEI2, and ACEI groups. Compared with the control group, serum ACE activity in the $\mathrm{L}$ or $\mathrm{L}+\mathrm{Hyd}$ groups did not change, but significantly decreased in rats treated with ACEI (L+ACEI1, L+ACEI2, and ACEI groups). In contrast, tissue ACE activities in the heart and aorta markedly increased in the L group after the first, fourth, and eighth weeks of treatment, while ACE activities in other tissues (lung, kidney, and 


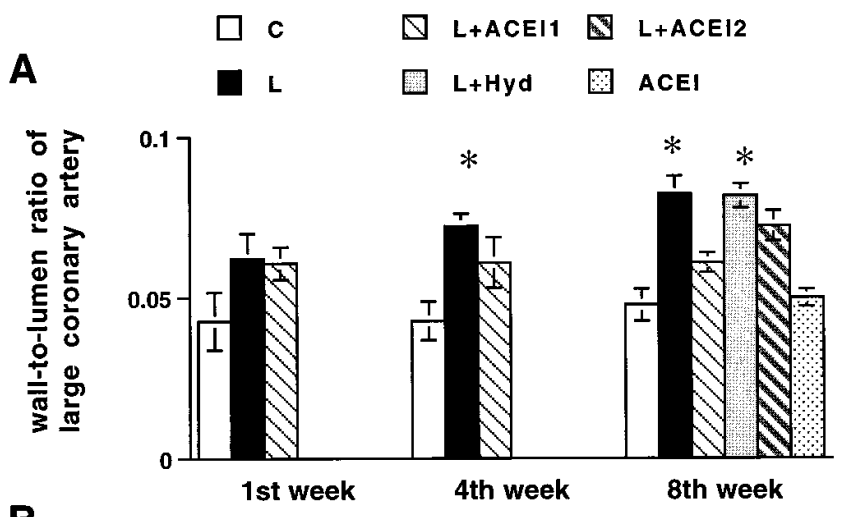

B

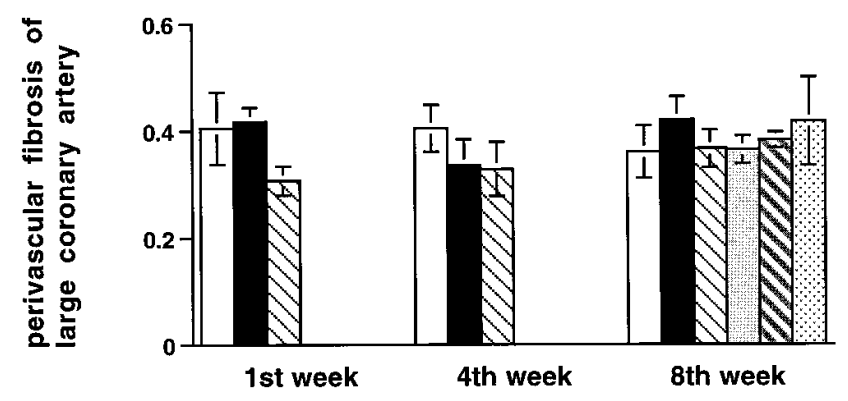

C

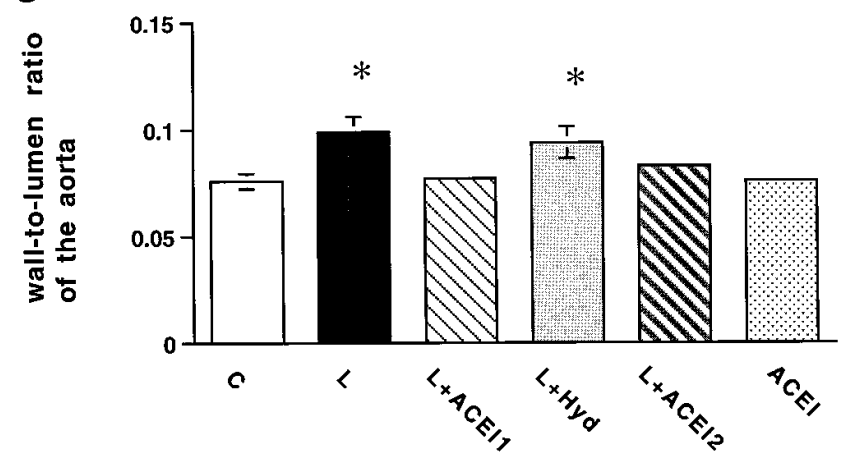

Figure 4. Wall-to-lumen ratio in large coronary arteries at the first, fourth, and eighth week of treatment $(A)$, perivascular fibrosis in large coronary arteries at the first, fourth, and eighth week of treatment $(B)$, and the wall-to-lumen ratio in the thoracic aorta at the eighth week of treatment $(C) .{ }^{*} P<0.01$ versus the $C$ group by twoway ANOVA and multiple comparison $t$ tests.

liver) were unchanged. The increases in ACE activities in cardiovascular tissues in the $\mathrm{L}$ group were blunted by both the low and high doses of ACEI, but not by hydralazine. Treatment with ACEI alone did not alter tissue ACE activity in the heart, but significantly decreased the ACE activity in all other tissues examined.

Relationships between the structural changes and the biochemical data at the eighth week of treatment were examined (Fig. 7). A significant correlation was found between the wallto-lumen ratio of the aorta and aortic ACE activity $(y=0.001 x$ $+0.075, r=0.62, P<0.01)$ and between the relative LV weight and cardiac ACE activity $(y=0.508 x+1.915, r=0.60$, $P<0.01)$. There was no significant correlation between the wall-to-lumen ratio of the aorta and the serum ACE $(P=0.74)$ or between the relative LV weight and the serum ACE $(P=$ $0.13)$. There was no significant correlation between the wallto-lumen ratio of the aorta and PRA $(P=0.33)$, or between the relative $\mathrm{LV}$ weight and PRA $(P=0.13)$.

In the control rats, ACE immunoreactivity was present in the endothelium of the large and small coronary arteries, but not in the vascular media and adventitia or in the myocardium. In the $\mathrm{L}$ and $\mathrm{L}+\mathrm{Hyd}$ groups, both the endothelium and the areas of perivascular fibrosis were intensely immunoreactive to ACE antibody (Fig. 8). No immunoreactivity was noted in the vascular media. The myocardial myocytes were occasionally positive for the ACE antibody (data not shown). No such abnormal ACE immunoreactivity was noted in the L+ACEI1 group that showed no evidence of vascular and myocardial structural changes (data not shown). No immunoreactivity was noted when ACE antibody was replaced with nonimmune IgG (negative control).

\section{Discussion}

The role of ACE in the vascular and myocardial structural changes induced by long-term administration of L-NAME has not been investigated. The major findings of this study were that: $(a)$ the tissue, but not the serum ACE activity was increased before such structural changes developed; $(b)$ the structural changes and local ACE activation were prevented by ACEI; and (c) vascular and myocardial structural changes were associated with an increase in immunodetectable ACE. We interpret these findings to suggest that an activation of the local ACE may contribute to the development of coronary vascular and myocardial structural changes in this model.

It has been shown in rats that the long-term administration of L-NAME decreases NO synthesis and causes arterial hypertension (14-21). Such long-term administration of this agent also causes vascular and myocardial remodeling in rats $(15,17-$ 21). In this study, we examined the temporal relationship between structural changes and ACE activity after L-NAME administration in this model. This study demonstrated that the structural changes in coronary arteries occur by the fourth week, and the myocardial hypertrophy occurs by the eighth week of L-NAME administration, while the increases in tissue ACE activity were evident as early as the first week. The latter findings agree with recent reports by Arnal et al., who showed that rats did not develop cardiac hypertrophy after the fourth week of L-NAME administration (17) and that a subset of rats developed cardiac hypertrophy with increased tissue ACE activity (21). Thus, activation of tissue ACE preceded the development of vascular and myocardial structural changes. Although we previously failed to demonstrate structural changes in large coronary arteries (21), significant increases in the wallto-lumen ratio were evident in both the large coronary arteries and aorta in the present study. Thus, long-term administration of L-NAME appears to cause structural changes in the large conduit vessels as well as in small arteries. The reasons for the different findings between this study and our previous one are unclear. However, the differences may be related to the fact that older rats (20 wk old) were used in this study while younger ones (8 wk old) were used previously (21).

We also investigated the effects of ACEI on the vascular and myocardial structural changes in this rat model. These structural changes were markedly reduced by the administration of ACEI but not of hydralazine. Furthermore, a low dose 

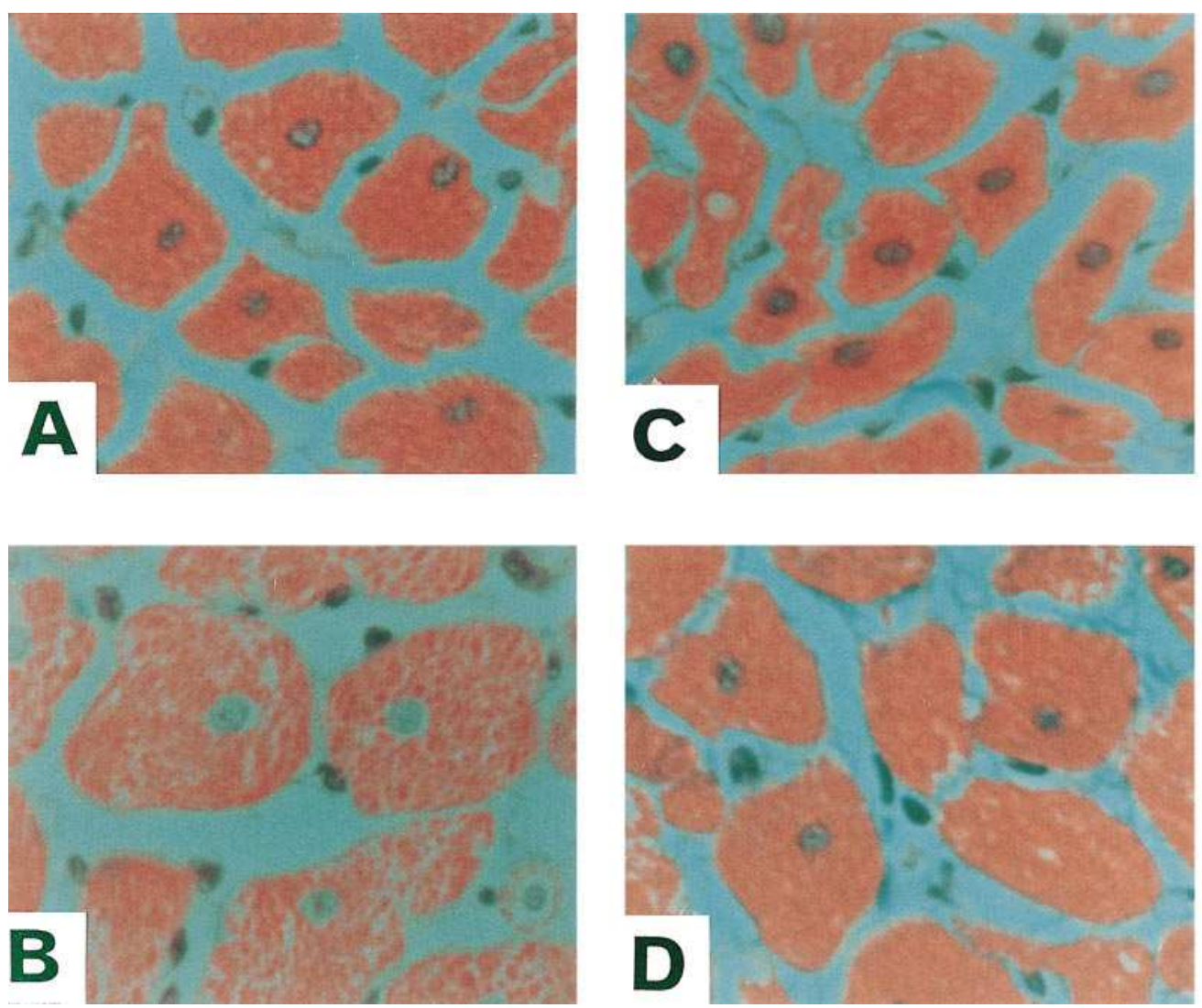

\section{$\overline{10} \mu \mathrm{m}$}

Figure 5. Micrographs of myocytes of LVs. Masson's trichrome stain in the control $(A), \mathrm{L}(B), \mathrm{L}-\mathrm{NAME}+\mathrm{ACEI}(C)$, and L-NAME+Hyd $(D)$ groups. A bar indicates $10 \mu \mathrm{m}$.

of ACEI, one that did not affect arterial pressure, produced inhibitory effects on the structural changes and tissue ACE activity identical to those seen with the high dose of ACEI. Thus, the inhibition of ACE, not the prevention of arterial hyperten- sion induced by L-NAME, appears to account for the beneficial effects of ACEI observed in this model. In addition, we evaluated the effects of ACEI on PRA in this model. PRA did not change after the first or fourth week, and increased after

Table II. Effects of Various Treatments on Biochemical Variables

\begin{tabular}{|c|c|c|c|c|c|c|c|}
\hline Parameter & Week & Control & $\begin{array}{l}\text { L-NAME } \\
\text { alone }\end{array}$ & $\begin{array}{l}\text { L-NAME } \\
+ \text { +Hyd }\end{array}$ & $\begin{array}{l}\text { L-NAME } \\
\text { +ACEI1 }\end{array}$ & $\begin{array}{l}\text { L-NAME } \\
\text { + ACEI2 }\end{array}$ & $\begin{array}{l}\text { ACEI } \\
\text { alone }\end{array}$ \\
\hline \multicolumn{8}{|l|}{ PRA } \\
\hline \multirow[t]{3}{*}{$(\mathrm{ng} / \mathrm{ml} / \mathrm{h})$} & 1 & $27 \pm 4$ & $26 \pm 3$ & NM & $47 \pm 3 *$ & NM & NM \\
\hline & 4 & $25 \pm 3$ & $24 \pm 3$ & NM & $47 \pm 7^{\ddagger}$ & NM & NM \\
\hline & 8 & $26 \pm 2$ & $46 \pm 7 *$ & $43 \pm 7 *$ & $40 \pm 4 *$ & $40 \pm 3^{\ddagger}$ & $54 \pm 7 *$ \\
\hline \multicolumn{8}{|c|}{ Serum ACE activity $(\mathrm{nmol} / \mathrm{ml} / \mathrm{h})$} \\
\hline & 1 & $0.8 \pm 0.1$ & $0.8 \pm 0.05$ & $\mathrm{NM}$ & $0.2 \pm 0.1 *$ & NM & NM \\
\hline & 4 & $0.8 \pm 0.05$ & $0.7 \pm 0.04$ & NM & $0.1 \pm 0.01 *$ & NM & NM \\
\hline & 8 & $0.8 \pm 0.03$ & $0.8 \pm 0.05$ & $0.8 \pm 0.1$ & $0.4 \pm 0.1^{*}$ & $0.2 \pm 0.03 *$ & $0.3 \pm 0.1 *$ \\
\hline \multicolumn{8}{|c|}{ Tissue ACE activity (nmol/mg/h) } \\
\hline \multirow[t]{3}{*}{ Heart } & 1 & $0.9 \pm 0.1$ & $1.6 \pm 0.1 *$ & $\mathrm{NM}$ & $0.6 \pm 0.2 *$ & NM & NM \\
\hline & 4 & $0.7 \pm 0.1$ & $1.5 \pm 0.1 *$ & NM & $0.3 \pm 0.02 *$ & $\mathrm{NM}$ & NM \\
\hline & 8 & $0.9 \pm 0.1$ & $1.7 \pm 0.1 *$ & $1.6 \pm 0.2^{\ddagger}$ & $0.6 \pm 0.1^{\ddagger}$ & $1.0 \pm 0.1$ & $1.0 \pm 0.02$ \\
\hline \multirow[t]{3}{*}{ Thoracic aorta } & 1 & $6.1 \pm 0.6$ & $8.6 \pm 0.9^{\ddagger}$ & NM & $0.5 \pm 0.1 *$ & NM & NM \\
\hline & 4 & $5.7 \pm 0.6$ & $18.3 \pm 1.0^{*}$ & NM & $0.6 \pm 0.1 *$ & NM & NM \\
\hline & 8 & $5.3 \pm 0.4$ & $22.7 \pm 1.5^{*}$ & $18.2 \pm 3.8^{*}$ & $0.8 \pm 0.1 *$ & $2.0 \pm 0.3^{\ddagger}$ & $0.9 \pm 0.1^{*}$ \\
\hline Lung & 8 & $280 \pm 20$ & $296 \pm 25$ & $309 \pm 6$ & $31 \pm 3 *$ & $35 \pm 3 *$ & $36 \pm 6^{*}$ \\
\hline Kidney & 8 & $1.9 \pm 0.1$ & $1.9 \pm 0.25$ & $1.7 \pm 0.15$ & $0.4 \pm 0.1^{*}$ & $0.3 \pm 0.06^{*}$ & $0.4 \pm 0.07 *$ \\
\hline Liver & 8 & $0.3 \pm 0.03$ & $0.3 \pm 0.02$ & $0.2 \pm 0.02$ & $0.1 \pm 0.04 *$ & $0.1 \pm 0.02 *$ & $0.1 \pm 0.02 *$ \\
\hline
\end{tabular}

Values are mean \pm SEM. ${ }^{*} P<0.01,{ }^{\ddagger} P<0.05$, versus the control group. $\mathrm{NM}=$ not measured. 


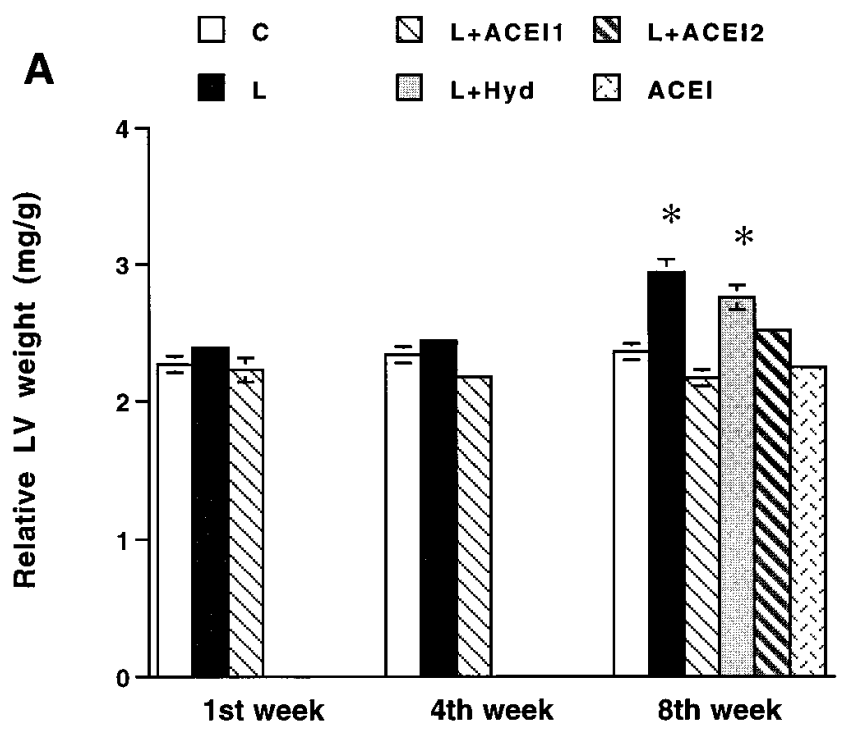

B

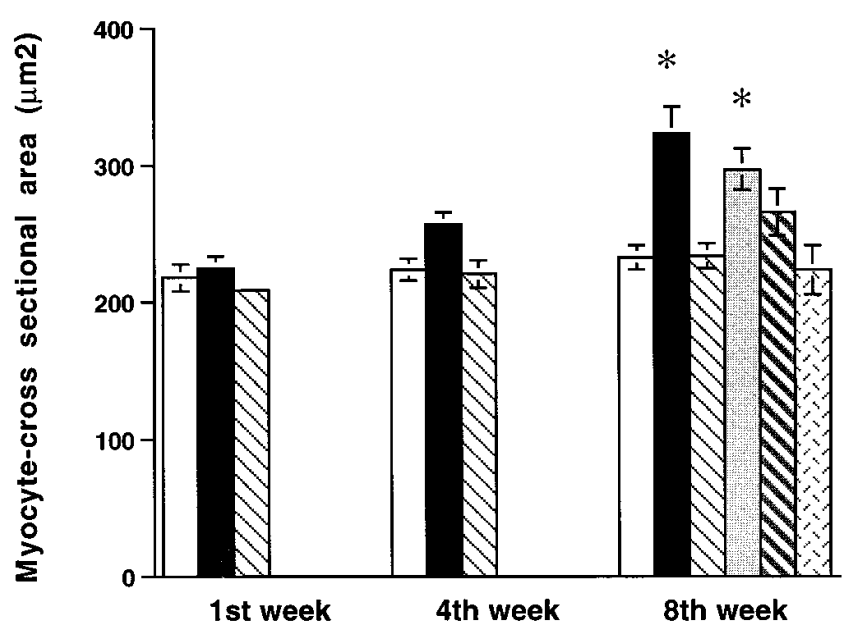

Figure 6. Relative LV weight $(A)$ and cross-sectional area of LV myocytes $(B)$ in the control, L, L+Hyd, L+ACEI1, L+ACEI2, and ACEI groups. $* P<0.01$ versus the control group by two-way ANOVA and multiple comparison $t$ tests.

the eighth week of L-NAME administration. These time-related changes in PRA are consistent with previous reports (17-22), and have been attributed to the nephroangiosclerosis seen in this model (18). It is possible that the increased PRA secondary to the nephroangiosclerosis would result in an increased synthesis of angiotensin II. This could occur even in the absence of tissue ACE activation and thus contribute to the cardiovascular structural changes. In this study, however, coronary vascular structural changes were evident before the increase in PRA was noted. There was no significant correlation between PRA and the vascular and myocardial structural changes.

These results strongly suggest that the activation of local ACE contributes to coronary vascular and myocardial structural changes in this model. However, the mechanisms by which ACE is activated after long-term administration of L-NAME were not explored in this study. It has been shown that NO inhibits vascular proliferation, and thus contributes to structural changes in the vascular wall (5-9). A decreased NO synthesis induces the synthesis of growth-promoting factors from the endothelium (33). The activation of ACE would increase the synthesis of angiotensin II, which in turn directly induces vascular smooth muscle proliferation (34) and myocyte hypertrophy $(23,24)$, and also induces the release of plateletderived growth factor (35) and transforming growth factor- $\beta$ (36). In addition, long-term administration of angiotensin II causes perivascular fibrosis in a rat model $(37,38)$. This angiotensin II-induced fibrosis resembles the histopathological findings seen in this study. In the present study, a significant correlation was demonstrated between the cardiovascular structural changes and tissue ACE activities. Tissue ACE activities were also increased only in the heart and arteries but not in other organs evaluated. The selective rise of ACE activities in cardiovascular tissues implies that ACE may have been activated locally in these tissues in this model. We thus examined the site of ACE activation using immunohistochemistry and found that the structural changes in the coronary arteries were characterized by an increase in immunodetectable ACE. These findings further substantiate the importance of the local synthesis of angiotensin II in myocyte hypertrophy, as reported by Sadoshima et al. (39). These findings also support the hypothesis that vascular and myocardial structural changes in this model are mediated by the local elaboration of growth-promoting factors, which in turn are induced by an activation of the renin-angiotensin system, including ACE.

It should be noted that long-term administration of L-NAME produces pathological vascular structural changes in other organs. Michel et al. (40) showed that L-NAME-induced vascular damages in the kidney were prevented by ACEI. Although renal histopathology was not examined in this study, these findings suggest a possibility that in the kidney L-NAMEinduced vascular structural changes can develop without the overall increase in tissue ACE activity.

There are two possible mechanisms by which ACEI exerted its beneficial effects on cardiovascular remodeling in this study. First, ACEI may specifically act on ACE to inhibit the formation of angiotensin II. Second, because ACE is identical to kininase II, its elevated presence may have inhibited the breakdown of bradykinin such that the resultant increase in NO release might have contributed to the beneficial effects of ACEI $(41,42)$. However, we did not investigate whether the beneficial cardiovascular effects of ACEI may have resulted from inhibition of either angiotensin II-induced actions or bradykinin breakdown.

This study has several implications. First, our data suggest a possible link between NO synthesis and tissue ACE in the development of vascular and myocardial disorders. Second, we suggested previously that endothelial dysfunction occurs in the coronary microcirculation of patients with angina pectoris and normal coronary arteriograms $(43,44)$, conditions in which coronary microvascular disorders contribute to myocardial ischemia (45). The marked thickening of the media of microvessels observed in the present study, using rats, resembles the histopathological findings in patients with microvascular angina (46). Kaski et al. (47) demonstrated that long-term administration of ACEI reduced the myocardial ischemia induced by stress in patients with microvascular angina. The present animal model may thus be relevant to the syndrome of microvascular angina seen clinically.

We conclude that an increase in local ACE expression is 
A
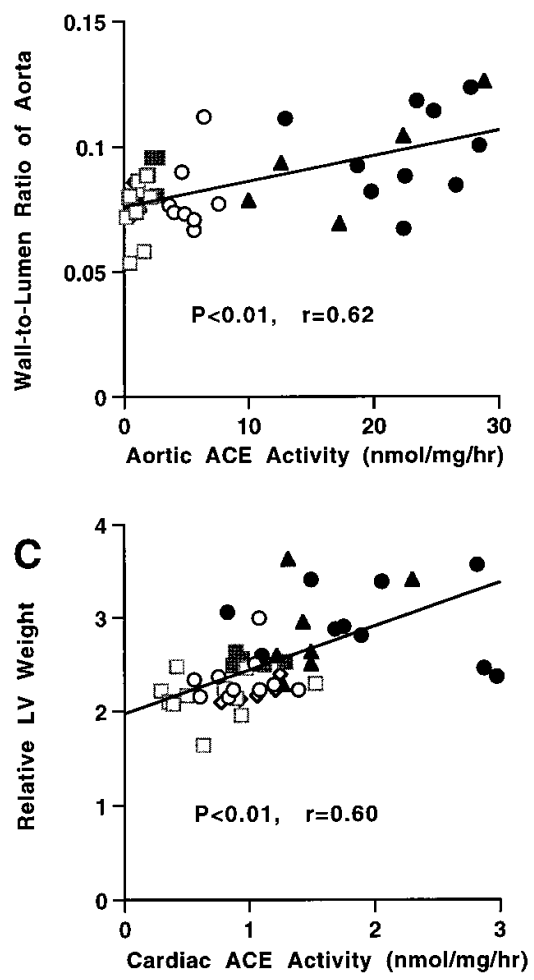

B
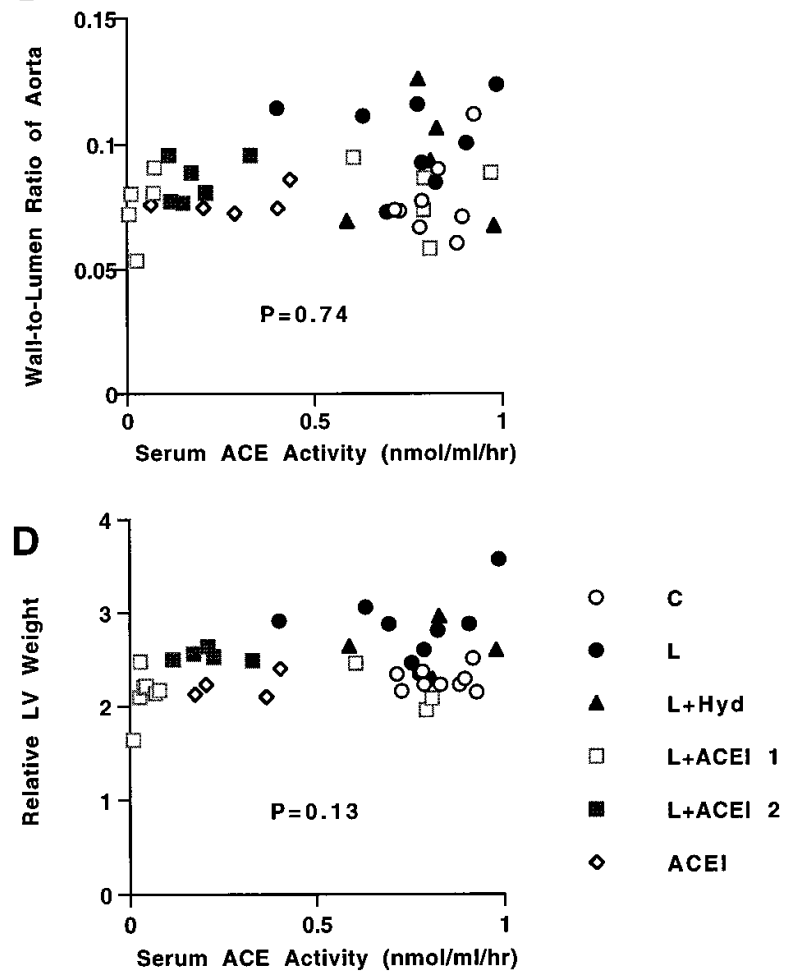

Figure 7. Scatter plots showing the relationship between structural changes and biochemical parameters. $(A)$ The wall-to-lumen ratio of aorta and aortic ACE activity. $(B)$ The wall-to-lumen ratio of aorta and serum ACE activity. (C) The relative $L V$ weight and cardiac ACE activity. $(D)$ The relative $L V$ weight and serum ACE activity in the control, L, L+Hyd, L+ACEI1, L+ACEI2, and ACEI groups.
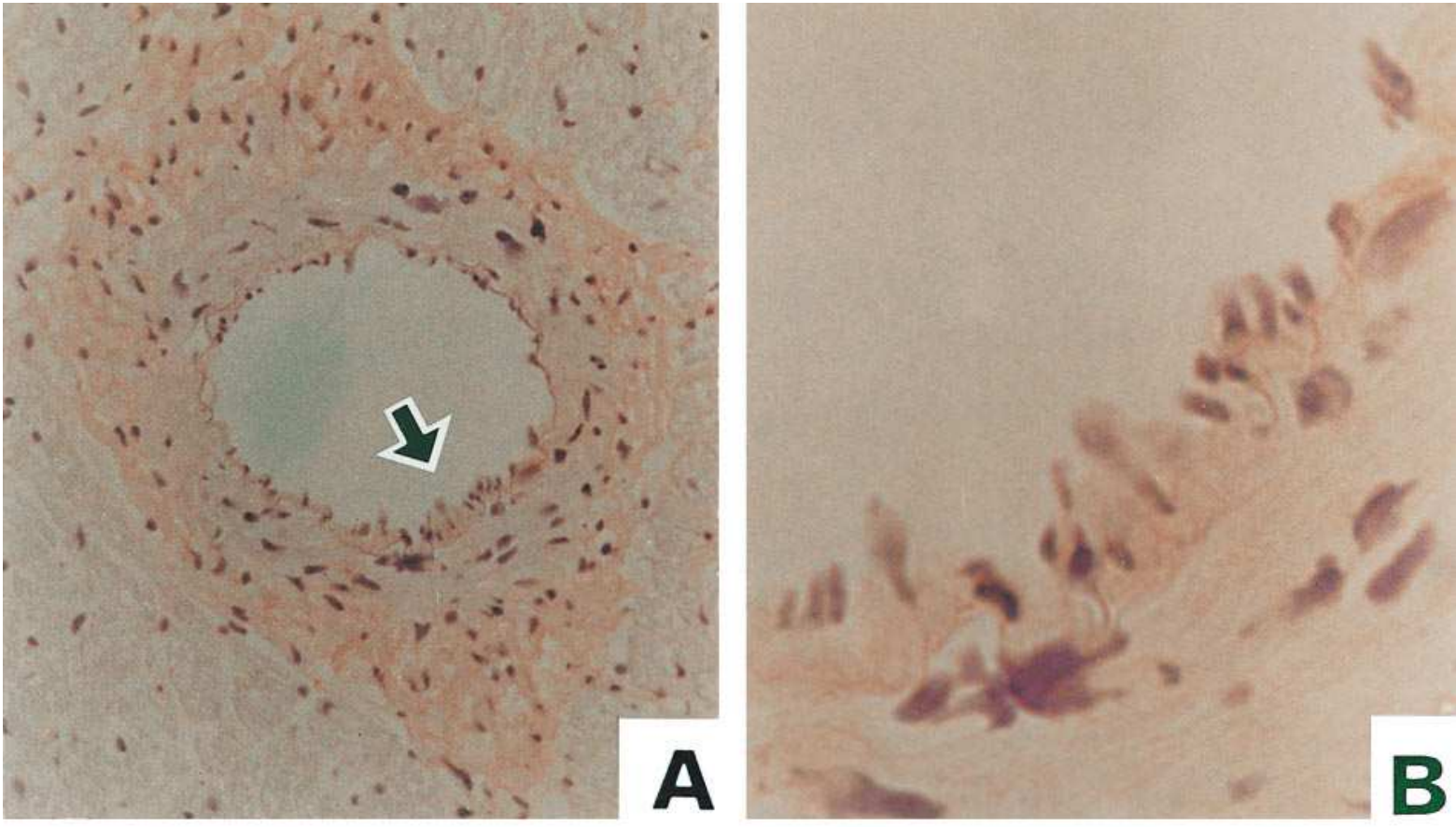

\section{$100 \mu \mathrm{m}$}

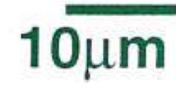

Figure 8. Micrographs of immunohistochemistry for ACE in the L group after the eighth week of treatment. $(A)$ An immunohistochemical picture of coronary artery. $(B)$ An expanded view of the area is indicated by the arrow in $A$. 
important in the pathogenesis of the vascular and myocardial remodeling induced by long-term blockade of NO synthesis in the rat model.

\section{Acknowledgments}

The authors thank Ms. Amano, Ms. Takebe, Mr. Fujii, and Ms. Noguchi for providing their excellent assistance.

This study was supported by Grants-in Aid for Scientific Research $(06670725,06404034$, and 07557346$)$ from the Ministry of Education, Science and Culture, Tokyo, and a Research Grant from the Uehara Memorial Foundation, Tokyo, Japan.

\section{References}

1. Moncada, S., and A. Higgs. 1993. The L-arginine-nitric oxide pathway. $N$. Engl. J. Med. 329:2002-2012.

2. Dzau, V.J., and G.H. Gibbons. 1991. Endothelium and growth factors in vascular remodeling of hypertension. Hypertension (Dallas). 18(Suppl. III): III115-III121.

3. Meyers, P.R., R.L. Minor, Jr., R. Guerra, Jr., J.N. Bates, and D.G. Harrison. 1990. Vasorelaxant properties of the endothelium-derived relaxing factor more closely resemble to $S$-nitrocysteine than nitric oxide. Nature (Lond.). 345: 161-163.

4. Palmer, R.M., A.G. Ferrige, and S. Moncada. 1987. Nitric oxide release accounts for the biological activity of endothelium-derived relaxing factor. $\mathrm{Na}$ ture (Lond.). 327:524-526.

5. Garg, U.C., and A. Hassid. 1989. Nitric oxide-generating vasodilators and 8-bromo-cyclic guanosine monophosphate inhibit mitogenesis and proliferation of cultured rat vascular smooth muscle cells. J. Clin. Invest. 83:1774-1777.

6. McNamara, D.B., B. Bedi, H. Aurora, L. Tena, L.J. Ignarro, P.J. Kadowitz, and D.J. Akers. 1993. L-arginine inhibits balloon catheter-induced intimal hyperplasia. Biochem. Biophys. Res. Commun. 193:291-296.

7. Cooke, J.P., A.H. Singer, P. Tsao, P. Zera, R.A. Rowan, and M.E. Billingham. 1992. Antiatherogenic effects of L-arginine in the hypercholesterolemic rabbit. J. Clin. Invest. 90:1168-1172.

8. Cayatte, A.J., J.J. Palacino, K. Horten, and R.A. Cohen. 1994. Chronic inhibition of nitric oxide production accelerates neointima formation and impairs endothelial function in hypercholesterolemic rabbits. Arterioscler. Thromb. 14:753-759.

9. Von der Leyen, H., G.H. Gibbons, R. Morishita, N.P. Lewis, L. Zhang, M. Nakajima, Y. Kaneda, J.P. Cooke, and V. Dzau. 1995. Gene therapy inhibiting neointimal vascular lesion: in vivo transfer of endothelial cell nitric oxide synthase gene. Proc. Natl. Acad. Sci. USA. 92:1137-1141.

10. Egashira, K., T. Inou, Y. Hirooka, A. Yamada, Y. Maruoka, H. Kai, M. Sugimachi, and A. Takeshita. 1993. Impaired coronary blood flow response to acetylcholine in patients with coronary risk factors and proximal atherosclerotic lesions. J. Clin. Invest. 91:29-37.

11. Zeiher, A.M., H. Drexler, B. Saubier, and H. Just. 1993. Endotheliummediated coronary blood flow modulation in humans. Effects of age, atherosclerosis, hypercholesterolemia, and hypertension. J. Clin. Invest. 92:652-662.

12. Kuo, L., M.J. Davis, M.S. Cannon, and W.M. Chillian. 1992. Pathophysiological consequences of atherosclerosis extend into the coronary microcirculation. Restoration of endothelium-dependent responses by L-arginine. Circ. Res. 70:465-476.

13. Egashira, K., T. Inou, Y. Hirooka, A. Yamada, Y. Maruoka, H. Kai, M. Sugimachi, S. Suzuki, and A. Takeshita. 1993. Impaired coronary blood flow response to acetylcholine in patients with atherosclerosis and proximal atherosclerotic lesions. J. Clin. Invest. 91:29-39.

14. Ito, A., K. Egashira, T. Kadokami, Y. Fukumoto, T. Takayanagi, R. Nakaike, T. Kuga, K. Sueishi, H. Shimokawa, and A. Takeshita. 1995. Chronic inhibition of endothelium-derived nitric oxide synthesis causes coronary microvascular structural changes and hyperreactivity to serotonin in pigs. Circulation. 92:2636-2644.

15. Numaguchi, K., K. Egashira, M. Takemoto, T. Kadokami, H. Shimokawa, K. Sueishi, and A. Takeshita. 1995. Chronic inhibition of nitric oxide synthesis causes coronary microvascular remodeling in rats. Hypertension (Dallas). 26(Part 1):957-962.

16. Kadokami, T., K. Egashira, K. Kuwata, Y. Fukumoto, T. Kozai, H. Yasutake, T. Kuga, H. Shimokawa, K. Sueishi, and A. Takeshita. 1996. Altered serotonin receptor subtypes contribute to microvascular hyperreactivity to serotonin in pigs with chronic inhibition of nitric oxide. Circulation. 94:182189.

17. Arnal, J.F., L. Warin, and J.B. Michel. 1992. Determinants of aortic cyclic guanosine monophosphate in hypertension induced by chronic inhibition of nitric oxide synthase. J. Clin. Invest. 90:647-652.

18. Baylis, C., B. Mitruka, and A. Deng. 1992. Chronic blockade of nitric oxide synthesis in the rat produces systemic hypertension and glomerular dam- age. J. Clin. Invest. 90:278-281.

19. Bank, N., H.S. Aynedjian, and G.A. Khan. 1994. Mechanism of vasoconstriction induced by chronic inhibition of nitric oxide in rats. Hypertension (Dallas). 24:322-328.

20. Ribeiro, M.O., E. Antunes, G. Nicci, S.M. Lovisolo, and R. Zatz. 1992 Chronic inhibition of nitric oxide synthesis. A new model of arterial hypertension. Hypertension (Dallas). 20:298-303.

21. Arnal, J.F., A.I.E. Amrani, G. Chatellier, J. Menard, and J.B. Michel. 1993. Cardiac weight in hypertension induced by nitric oxide synthase blockade. Hypertension (Dallas). 22:380-387.

22. Schieffer, B., A. Wirger, M. Meybrunn, S. Seitz, J. Holtz, U.N. Riede, and H. Drexler. 1994. Comparative effects of angiotensin-converting enzyme inhibition and angiotensin II type 1 receptor blockade on cardiac remodeling after myocardial infarction in the rat. Circulation. 89:2273-2282.

23. Weinberg, E.O., F.J. Schoen, D. George, Y. Kagaya, P.S. Douglas, S.E Litwin, H. Schunkert, C.R. Benedict, and B.H. Lorell. 1994. Angiotensin-converting enzyme inhibition prolongs survival and modifies the transition to heart failure in rats with pressure overload hypertrophy due to ascending aortic stenosis. Circulation. 90:1410-1422.

24. Schunkert, H., V.J. Dzau, S.S. Tan, A.T. Hirsch, C.S. Apstein, and B.H. Lorell. 1990. Increased rat cardiac angiotensin converting enzyme activity and mRNA expression in pressure overload left ventricular hypertrophy: effects on coronary resistance, contractility and relaxation. J. Clin. Invest. 86:1913-1920.

25. Hirsch, A.T., H. Schunkert, M. Paul, and V.J. Dzau. 1991. Tissue-specific activation of cardiac angiotensin converting enzyme in experimental heart failure. Circ. Res. 69:475-482.

26. Schunkert, H., J.R. Ingelfinger, A.T. Hirsch, S.S. Tang, S.E. Litwin, C.E. Talsness, and V.J. Dzau. 1992. Evidence for tissue-specific activation of renal angiotensin mRNA expression in chronic stable experimental heart failure. $J$. Clin. Invest. 90:1523-1529.

27. The SOLVD Investigators. 1991. Effect of enalapril on survival in patients with reduced left ventricular ejection fractions and congestive heart failure. N. Engl. J. Med. 325:293-302.

28. Swedberg, K., P. Held, J. Kjekshus, K. Rasmossen, L. Ryden, and H Wedel. 1992. CONSENSUS II: effects of the early administration of enalapril on mortality in patients with acute myocardial infarction. N. Engl. J. Med. 327: $678-684$.

29. Loud, A.V., P. Anversa, F. Giacomelli, and J. Wiener. 1978. Absolute morphometric study of myocardial hypertrophy in experimental hypertension. I. Determination of myocyte size. Lab. Invest. 38:586-596.

30. Anversa, P., A.V. Loud, F. Giacomelli, and J. Wiener. 1978. Absolute morphometric study of myocardial hypertrophy in experimental hypertension. II. Ultrastructure of myocytes and interstitium. Lab. Invest. 38:597-609.

31. Cheung, H.S., and D.W. Cushman. 1973. Inhibition of homogenous angiotensin-converting enzyme of rabbit lung by synthetic venom peptides of $\mathrm{Bo}$ throps jararaca. Biochem. Biophys. Acta. 293:451-463.

32. Hayashi, K., K. Nunami, J. Kato, N. Yoneda, M. Kubo, T. Ochiai, and R. Ishida. 1989. Studies of angiotensin-converting enzyme inhibitors. Synthesis and angiotensin-converting enzyme inhibitory activities of 3-acyl-1-alkyl-2-oxoimidazolidine-4-carboxylic acid derivatives. J. Med. Chem. 32:289-297.

33. Kourembanas, S., L.P. McQuillan, G.K. Leung, and D.V. Faller. 1993. Nitric oxide regulates the expression of vasoconstrictors and growth factors by vascular endothelium under both normoxia and hypoxia. J. Clin. Invest 92:99104

34. Itoh, H., M. Mukoyama, R.E. Pratt, G.H. Gibbons, and V.J. Dzau. 1993. Multiple autocrine growth factors modulate vascular smooth muscle cell growth response to angiotensin II. J. Clin. Invest. 91:2268-2274.

35. Naftilan, A.J., R.E. Pratt, and V.J. Dzau. 1989. Induction of plateletderived growth factor A chain and c-myc gene expressions by angiotensin II in cultured rat vascular smooth muscle cells. J. Clin. Invest. 83:1419-1424.

36. Gibbons, G.H., R.E. Pratt, and V.J. Dzau. 1992. Vascular smooth muscle cell hypertrophy vs. hyperplasia: autocrine transforming growth factor-beta 1 expression determines growth response to angiotensin II. J. Clin. Invest. 90: 456-461.

37. Sigusch, H.H., S.E. Campbell, and K.T. Weber. 1996. Angiotensin IIinduced myocardial fibrosis in rats: role of nitric oxide, prostaglandins and bradykinin. Cardiovasc. Res. 31:546-554

38. Hou, J., H. Kato, R.A. Cohen, A.V. Chobanian, and P. Brecher. 1995. Angiotensin II-induced cardiac fibrosis in rats is increased by chronic inhibition of nitric oxide synthase. J. Clin. Invest. 96:2469-2477.

39. Sadoshima, J., Y. Xu, H.S. Slayter, and S. Izumo. 1993. Autocrine release of angiotensin II mediates stretch-induced hypertrophy of cardiac myocytes in vitro. Cell. 75:977-984.

40. Michel, J.B., Y. Xu, S. Blot, M. Philippe, and G. Chatellier. 1996. Improved survival in rats administered NG-nitro-L-arginine methyl ester due to converting enzyme inhibitor. J. Cariovasc. Pharmacol. 28:142-148.

41. Farhy, R.D., O.A. Carretero, K.L. Ho, and A.G. Scicli. 1993. Role of kinins and nitric oxide in the effects of angiotensin converting enzyme inhibitors on neointimal proliferation. Circ. Res. 72:1202-1210.

42. deBlois, D., D.M. Lombardi, M.A. Garvin, and S.M. Schwartz. 1992. Inhibition by ramipril of intimal hyperplasia in the denuded rat carotid is reversed by HOE140, a kinin B2 receptor antagonist. Circulation. 86:I-226 (Abstr.). 
43. Egashira, K., T. Inou, Y. Hirooka, A. Yamada, Y. Urabe, and A. Takeshita. 1993. Evidence of impaired endothelium-dependent coronary vasodilation in patients with angina pectoris and normal coronary angiograms. $N$. Engl. J. Med. 328:1659-1664.

44. Egashira, K., Y. Hirooka, T. Kuga, M. Mohri, and A. Takeshita. 1996. Effects of L-arginine supplementation on endothelium-dependent coronary vasodilation in patients with angina pectoris and normal coronary arteriograms. Circulation. 94:130-134.

45. Maseri, A., F. Crea, and J.C. Kaski. 1991. Mechanisms of angina pecto- ris in syndrome X. J. Am. Coll. Cardiol. 17:499-506.

46. Mosseri, M., R. Yarom, M.S. Gostman, and Y. Hasin. 1986. Histologic evidence for small-vessel coronary artery disease in patients with angina pectoris and patent large coronary arteries. Circulation. 74:964-972.

47. Kaski, J.C., G. Rosano, S. Gavrielides, and L. Chen. 1994. Effects of angiotensin-converting enzyme inhibition on exercise-induced angina and ST segment depression in patients with microvascular angina. J. Am. Coll. Cardiol. 23: $652-657$. 